\title{
Satisfacción laboral de la enfermería en las prisiones españolas
}

\author{
EJ Vera-Remartínez ${ }^{1}$, LM Mora Parra², JA González Gómez ${ }^{3}$, \\ J García Jiménez ${ }^{3}$, E Garcés Pina ${ }^{4}$, JA Domínguez Zamorano ${ }^{5}$, \\ JR Borraz Fernández ${ }^{6}$, A Blanco Quiroga ${ }^{7}, B$ Armenteros López ${ }^{8}$
}

\author{
${ }^{1}$ Centro Penitenciario de Castellón 1 \\ ${ }^{2}$ Centro Penitenciario de Madrid 2 \\ ${ }^{3}$ Centro Penitenciario de Murcia \\ ${ }^{4}$ Centro Penitenciario de Zuera (Zaragoza) \\ ${ }^{5}$ Centro Penitenciario de Huelva \\ ${ }^{6}$ Centro Penitenciario del Dueso (Santander) \\ ${ }^{7}$ Centro Penitenciario de Brieva (Ávila) \\ ${ }^{8}$ Centro Penitenciario de Quatre Camins (Barcelona) \\ (Grupo de Enfermería de la Sociedad Española de Sanidad Penitenciaria)
}

\section{RESUMEN}

Fundamento: No existen trabajos que valoren la satisfacción laboral de los profesionales de enfermería en las prisiones españolas. Pretendemos averiguar el nivel de satisfacción global y de cada uno de sus componentes.

Método: Estudio descriptivo transversal y multicéntrico realizado en las prisiones españolas. Se utiliza el cuestionario de satisfacción Font Roja, adaptado por J. Arranz, que mide la satisfacción laboral con una escala de Likert. Utilizamos pruebas paramétricas y se construye un modelo de regresión con fines predictivos.

Resultados: Respondieron al cuestionario 376 enfermeras (Tasa de Participación 62,7\%; Tasa de Respuesta del 76,7\%). Participaron 67 centros $(91,8 \%$ ). La puntuación media de la satisfacción es de 2,84 (IC 95\%: 2,81-2,87). Las dimensiones menos valoradas han sido variedad de la tarea 1,66 (I.C. 95\%: 1,58-1,74); presión en el trabajo 2,15 (I.C. 95\%: 2,08-2,23) y control sobre el trabajo 2,77 (I.C. 95\%: 2,73-2,82). La puntuación más alta fue para la satisfacción en el trabajo 3,52 (I.C. 95\%: 3,44-3,58).

Conclusiones: El índice medio de satisfacción laboral fue menor que en otros colectivos extra-penitenciarios. Se necesitan medidas correctoras.

Palabras clave: Enfermería; Prisiones; Satisfacción Laboral; Estudio Multicéntrico; Trabajo; Personal de Enfermería.

\section{JOB SATISFACTION OF NURSING STAFF IN SPANISH PRISONS}

\section{ABSTRACT}

Background: There are no available studies assessing job satisfaction amongst nursing staff in Spanish prisons. The aim of this study is to establish overall levels of job satisfaction and determine each of the components.

Method: Cross-sectional and multi-centre descriptive study conducted in Spanish prisons. A Font Roja satisfaction questionnaire adapted by J. Arranz for the study was used to measure degrees of job satisfaction using a Likert's scale. A parametric test was used and a regression model was constructed for predictive ends.

Results: 376 nurses answered the questionnaire (Participation Rate 62.7\%; Response Rate 76.7\%) 67 centres took part (91.8\%). The average satisfaction mark was 2.84 (CL 95\%: 2.81-2.87). The lowest ranked components were job variety 1.66 (CL 95\%: 1.58-1.74), job-related stress 2.15 (CL 95\%: 2.08-2.23) and control over job 2.77 (CL 95\%: 2.73-2.82). The highest ranked aspect was job satisfaction, averaging 3.52 (CL 95\%: 3.44-3.58).

Conclusions: The average satisfaction mark for prison nursing staff was low when compared to other groups of health care professionals, which implies the need for corrective measures.

Key words: Nursing; Prisons; Job Satisfaction; Multicenter Study; Work; Nursing Staff. 


\section{INTRODUCCIÓN}

La labor realizada por los profesionales de enfermería de la Sanidad Penitenciaria es poco conocida y valorada, pero esencial en la prestación de cuidados a una población, que por sus características propias, la hacen exclusiva.

Aparte de las funciones propiamente asistenciales y de prestación de cuidados, se unen otra serie de funciones de prevención y promoción de salud, de higiene y saneamiento ambientales, gracias a las cuales, entre otras, se puede hablar actualmente de una asistencia sanitaria en las prisiones con un adecuado grado de calidad asistencial, entendiendo por ella, la medida en que los servicios de salud aumentan la probabilidad de obtener los deseados resultados de salud para el individuo y la población en consistencia con el conocimiento profesional de ese momento ${ }^{1}$.

El concepto de calidad asistencial incluye una serie de componentes como son la equidad, la efectividad, la accesibilidad, la eficiencia, la satisfacción del paciente y la satisfacción del profesional ${ }^{2}$.

La importancia del concepto de satisfacción del profesional reside, desde la óptica de la gestión de servicios sanitarios, en que el grado de calidad de los servicios ofertados en un sistema sanitario está directamente relacionado con el nivel de satisfacción de los profesionales que trabajan en él y en su vinculación con la motivación ${ }^{3}$.

La implantación de un modelo de calidad total requiere, por tanto, conocer la satisfacción laboral de los trabajadores. Aunque en términos de calidad de servicios no está tan clara su relación directa con la calidad de la atención, sí parece claro que al medir la satisfacción profesional se está comprobando la calidad de la organización y de sus servicios internos ${ }^{4}$.

La no existencia de estudios sobre la satisfacción profesional de enfermería en el ámbito penitenciario español, hace que este estudio tenga un mayor interés, y nuestra intención con el mismo, es de poder conocer de primera mano, cual es la situación actual al respecto. Nuestros objetivos principales son por tanto, valorar la satisfacción de los profesionales de la enfermería penitenciaria en su trabajo y describir la posible influencia sobre ésta satisfacción laboral de otras variables analizadas.

\section{MATERIAL Y MÉTODO}

Diseño: Se trata de un estudio descriptivo, transversal y multicéntrico, mediante la cumplimentación de un cuestionario.
Periodo de estudio: Fue realizado entre el día 15 de octubre de 2007 y el 10 de febrero de 2008.

Población: La población de referencia fue la constituida por todos los enfermeros/as que desarrollan su actividad profesional en centros penitenciarios españoles incluidos los de Cataluña, a pesar de tener trasferidas las competencias en materia penitenciaria, concretamente 600.

Establecemos como único criterio de inclusión, que de forma voluntaria deseen participar en el mismo.

Instrumentos de medida y procedimiento: Para la recogida de información se diseñó un cuestionario, y se consideró que el método de encuesta por correo podría originar un sesgo en la respuesta que desvirtuase los resultados, por lo que se optó por la entrega personal en cada centro, encargando a una persona responsable de la distribución y recogida de los mismos, siendo autocumplimentado por cada uno de los participantes.

Para la recogida de información sobre la satisfacción en el trabajo, se utilizó el cuestionario validado de Font Roja y adaptado por J. Aranaz ${ }^{5}$, este consta de 24 apartados agrupados en 7 factores que intentan explorar todas las áreas definidas como componentes de la satisfacción laboral:

- Factor 1: "Satisfacción en el trabajo". Contiene aquellos apartados que denotan que el trabajador se encuentra satisfecho con lo que hace, tiene la idea de que está siendo útil y de que obtiene reconocimiento por lo que hace.

- Factor 2: "Presión en el trabajo". Hace referencia a la sensación que el trabajador tiene con respecto a la relación tiempo y carga de trabajo.

- Factor 3: "Relación personal”. Se refiere al nivel de satisfacción que provoca en el trabajador la calidad y cantidad de relaciones sociales en el trabajo.

- Factor 4: "Distensión en el trabajo". Capacidad para desconectar del trabajo, competitividad, salario y sensación de poder hacer la tarea.

- Factor 5: “Adecuación para el trabajo". Sensación que el trabajador posee de estar plenamente capacitado y desempeñar el trabajo que por condición y preparación merece.

- Factor 6: "Control sobre el trabajo". Posibilidad de organizar el propio trabajo, aprender cosas nuevas y no verse alterado en el desempeño de su profesión.

- Factor 7: "Variedad de la tarea". Sensación que puede tener el trabajador de que el trabajo no varía de un día para otro. 
Las respuestas que se ofrecen al conjunto de preguntas de cada factor siguen una escala de Likert. La puntuación obtenida en cada factor será el equivalente a la suma simple de las puntuaciones de los apartados que lo componen, dividido por el número de apartados. Cada uno de ellos tiene valores que oscilan del 1 al 5, de tal modo que las mayores puntuaciones denotan mayor satisfacción.

Previamente al envío de los cuestionarios se realizó una prueba piloto con 30 sujetos similares a los que constituirían la muestra, para comprobar que el cuestionario funcionaba correctamente.

Variables recogidas: las podemos agrupar en dos tipos, variables descriptivas de la muestra (edad, sexo, centro de trabajo, años experiencia laboral y penitenciaria, formación continuada, realización de consultas de enfermería) y variables necesarias para la consecución de los objetivos (obtenidas del cuestionario adaptado de Font Roja, que constituyen las 7 dimensiones comentadas de la satisfacción).

Procesamiento de la información: Se creó una base de datos en la aplicación ACCESS-2003, programándose una introducción protegida de los datos mediante la codificación previa de los campos de respuesta para depurar errores en su introducción.

Una vez cumplimentada la base de datos, estos, se exportaron a un archivo de datos para ser procesados con el programa estadístico SPSS versión 12.

Una vez exportados, y previamente a la materialización de cualquier tipo de cálculo, se procedió a realizar un nuevo proceso de depuración de datos, para controlar la calidad de los mismos mediante cuatro tipos de técnicas:

- Pruebas lógicas y de rango.

- Pruebas de consistencia entre los datos.

- Obtención de distribuciones de valores "missing" o valores desconocidos de todas las variables mediante el procedimiento MVA (Missing Value Análisis) que lleva implementada la versión 12 de SPSS.

- Obtención de distribuciones de las variables del estudio para detectar valores improbables.

Análisis estadístico: se realizó en tres etapas:

1.- Análisis univariante: considerando para las variables cuantitativas: medias con sus correspondientes intervalos de confianza al 95\%, y para las variables cualitativas: frecuencias absolutas y relativas.

2.- Análisis bivariante: donde realizamos comparación de medias mediante la utilización de pruebas paramétricas como la de la T-Student, análisis de la varianza (ANOVA) y aplicación de correlación de Pearson ( $r$ ) entre variables cuantitativas.
3.- Análisis multivariante: estableciendo un modelo de regresión lineal múltiple y considerando como variable dependiente del modelo, la variable numérica, satisfacción global, del cuestionario de satisfacción laboral de Font Roja, e introduciendo otra serie de variables independientes, con la intención de generar un modelo con fines predictivos.

\section{RESULTADOS}

De la población total de estudio (600 profesionales), hubo 114 negativas $(19,0 \%)$ y con 110 de ellos no fue posible el contacto $(18,8 \%)$, resultando una muestra de estudio de 376 profesionales de enfermería, con una Tasa de Participación del 62,7\% y una Tasa de Respuesta de 76,7\%. Participaron enfermeros de 67 centros penitenciarios, lo que supone un $91,8 \%$ del total de centros del país.

De los 376 profesionales de enfermería 152 $(40,4 \%$; I.C. $95 \%: 35,4$ a 45,6$)$ fueron varones y 224 $(59,6 \%$; I.C. $95 \%: 54,4$ a 64,6$)$ fueron mujeres. La edad media del total de la muestra fue de 38,1 años (I.C. al 95\%: 37,3 a 39,0). Por sexos los varones presentaron una edad media de 40,4 años (I.C. al 95\%: 39,1 a 41,8 ) y para las mujeres de 36,5 años ( I.C. al

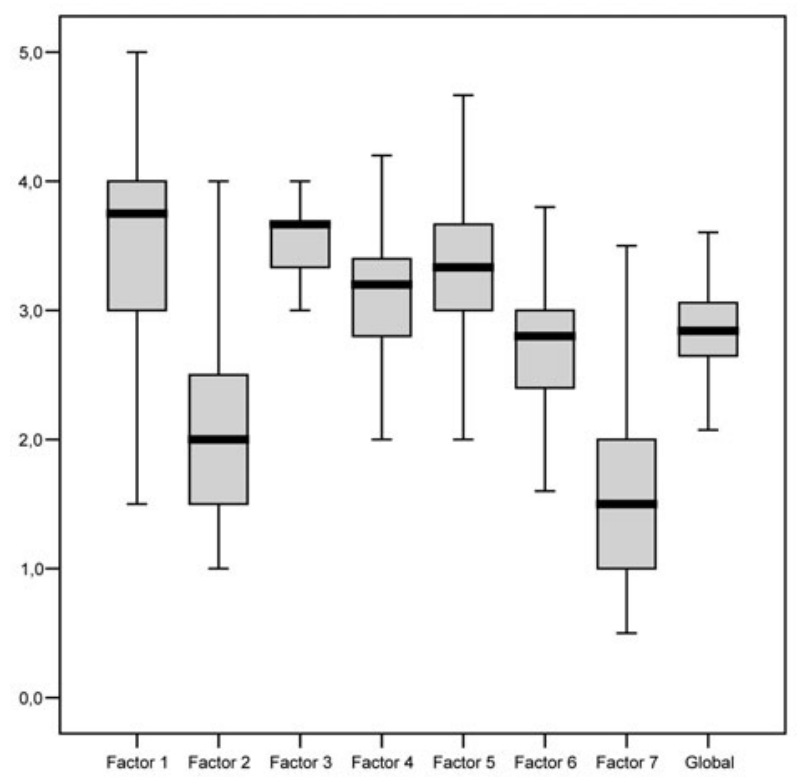

Factor 1 = Satisfacción en el trabajo. Factor 2 = Presión en el trabajo. Factor 3 = Relación personal. Factor $4=$ Distensión en el trabajo. Factor 5 = Adecuación para el trabajo. Factor $6=$ Control sobre el trabajo. Factor 7 = Variedad de la tarea. Global $=$ Índice general de satisfacción.

Figura 1: Puntuación media obtenida en las distintas dimensiones del cuestionario Font Roja. 


\begin{tabular}{|c|c|c|c|}
\hline & $\begin{array}{c}\text { Total de la muestra: } \\
\text { Media } \\
\text { (I.C. al } 95 \% \text { ) }\end{array}$ & $\begin{array}{c}\text { Mujeres: } \\
\text { Media } \\
\text { (I.C. al } 95 \% \text { ) }\end{array}$ & $\begin{array}{c}\text { Hombres: } \\
\text { Media } \\
\text { (I.C. al 95\%) }\end{array}$ \\
\hline Factor 1: Satisfacción en el trabajo & $\begin{array}{c}3,52 \\
(3,44 \text { a } 3,58)\end{array}$ & $\begin{array}{c}3,57^{*} \\
(3,48 \text { a } 3,67)\end{array}$ & $\begin{array}{c}3,43 \\
(3,32 \text { a } 3,54)\end{array}$ \\
\hline Factor 2: Presión en el trabajo & $\begin{array}{c}2,15 \\
(2,08 \text { a } 2,23)\end{array}$ & $\begin{array}{c}2,23^{*} \\
(2,14 \text { a } 2,32)\end{array}$ & $\begin{array}{c}2,04 \\
(1,91 \text { a } 2,17)\end{array}$ \\
\hline Factor 3: Relaciones personales & $\begin{array}{c}3,48 \\
(3,44 \text { a } 3,53)\end{array}$ & $\begin{array}{c}3,54 * \\
(3,48 \text { a } 3,60)\end{array}$ & $\begin{array}{c}3,40 \\
(3,31 \text { a } 3,48)\end{array}$ \\
\hline Factor 4: Distensión en el trabajo & $\begin{array}{c}3,08 \\
(3,24 \text { a } 3,13) \\
\end{array}$ & $\begin{array}{c}3,12 \\
(3,06 \text { a } 3,17)\end{array}$ & $\begin{array}{c}3,03 \\
(2,96 \text { a } 3,11) \\
\end{array}$ \\
\hline Factor 5: Adecuación para el trabajo & $\begin{array}{c}3,24 \\
(3,18 \text { a } 3,30)\end{array}$ & $\begin{array}{c}3,26 \\
(3,18 \text { a } 3,33)\end{array}$ & $\begin{array}{c}3,20 \\
(3,10 \text { a } 3,30)\end{array}$ \\
\hline Factor 6: Control sobre el trabajo & $\begin{array}{c}2,77 \\
(2,73 \text { a } 2,82) \\
\end{array}$ & $\begin{array}{c}2,75 \\
(2,69 \text { a } 2,81) \\
\end{array}$ & $\begin{array}{c}2,80 \\
(2,74 \text { a } 2,87) \\
\end{array}$ \\
\hline Factor 7: Variedad de la tarea & $\begin{array}{c}1,66 \\
(1,58 \text { a } 1,749)\end{array}$ & $\begin{array}{c}1,66 \\
(1,56 \text { a } 1,76)\end{array}$ & $\begin{array}{c}1,66 \\
(1,54 \text { a } 1,79)\end{array}$ \\
\hline Índice Global Satisfacción Laboral & $\begin{array}{c}2,84 \\
(2,81 \text { a } 2,87)\end{array}$ & $\begin{array}{c}2,88^{*} \\
(2,84 \text { a } 2,91)\end{array}$ & $\begin{array}{c}2,80 \\
(2,75 \text { a } 2,84)\end{array}$ \\
\hline
\end{tabular}

I.C. = Intervalo de Confianza. Resultados de la prueba de la T-Student (comparación de medias). Cuando la diferencia de medias es significativa el valor más alto se muestra con la significación $* \mathrm{p}<0,05 ; * \mathrm{p}<0,001$

Tabla 1: Puntuaciones medias en las distintas dimensiones del cuestionario de satisfacción laboral para el total de la muestra y por sexos.

95\%: 35,5 a 37,6), siendo la diferencia media de edad de 4 años, mayor para los varones $(p<0,0001)$.

El análisis descriptivo de las 7 dimensiones del cuestionario Font Roja (Figura 1) reveló puntuaciones bajas en las dimensiones: Variedad de la tarea 1,66 (I.C. 95\%: 1,58 a 1,74); Presión en el trabajo 2,15 (I.C. $95 \%$ : 2,08 a 2,23$)$ y control sobre el trabajo 2,77 (I.C. $95 \%: 2,73$ a 2,82 ). Las puntuaciones fueron altas en distensión para el trabajo 3,08 (I.C. 95\%: 3,04 a 3,13); adecuación para el trabajo 3,24 (I.C. $95 \%$ : 3,18 a 3,30); relaciones personales 3,48 (I.C. $95 \%: 3,44$ a 3,53 ) y satisfacción en el trabajo 3,52 (I.C. $95 \%$ : $3,44$ a 3,58$)$. Por sexos se obtienen puntuaciones ligeramente superiores en las mujeres para satisfacción en el trabajo, presión en el trabajo, relaciones personales y en el índice global (Tabla 1).

El índice global de satisfacción según el cuestionario Font Roja que encontramos tuvo un valor medio de 2,84 (I.C. $95 \% ; 2,81$ a 2,87 ) y un recorrido que va desde 2,00 a 3,60; su distribución fue normal ( $\mathrm{Z}$ de Kolmogorov-Smirnov $=0515 ; \mathrm{p}=0,954)$. En la Tabla 2 se adjuntan las medias obtenidas en los 24 ítems del test.

Por Comunidades Autónomas obtenemos una puntuación media más alta en Melilla, Ceuta y Navarra; y las puntuaciones más bajas corresponden a
Murcia, Asturias y Extremadura con diferencias estadísticamente significativas ( $\mathrm{p}=0,001)$. Ver Figura 2 .

En el análisis bivariante entre alguna de las características de los profesionales y la satisfacción glo-

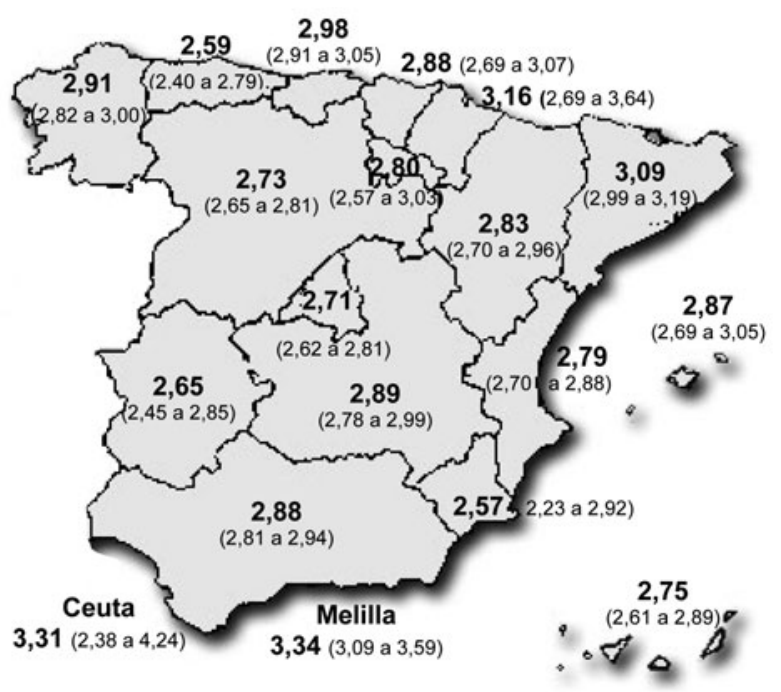

Figura 2: Distribución por CC.AA. de las medias del Índice de Satisfacción Profesional con su correspondiente Intervalo de Confianza al $95 \%$. 


\begin{tabular}{|c|c|c|c|}
\hline Preguntas: & Media & I.C. al 95\% & Dimensión: \\
\hline 1.- Mi trabajo no varía, resulta monótono & 3,65 & 3,54 a 3,76 & Variedad \\
\hline 2.- Tengo responsabilidad a la hora de tomar decisiones & 3,57 & 3,47 a 3,66 & Control \\
\hline 3.- Al final de la jornada me encuentro muy cansado & 3,27 & 3,18 a 3,36 & Control \\
\hline 4.- No desconecto del trabajo cuando llego a casa & 3,77 & 3,67 a 3,86 & Control \\
\hline 5.- En el trabajo diario me tengo que emplear a fondo & 3,48 & 3,40 a 3,56 & Control \\
\hline 6.- Mi trabajo me altera el estado de ánimo & 2,93 & 2,81 a 3,05 & Control \\
\hline 7.- En mi trabajo me encuentro satisfecho & 3,18 & 3,06 a 3,29 & Satisfacción \\
\hline 8.- Tengo independencia para organizar mi trabajo & 3,51 & 3,40 a 3,62 & Distensión \\
\hline 9.- Tengo oportunidades de aprender cosas nuevas & 2,46 & 2,36 a 2,56 & Distensión \\
\hline 10.- Tengo interés por las cosas que realizo & 3,34 & 3,24 a 3,43 & Satisfacción \\
\hline 11.- Tengo la sensación de que lo que hago no vale la pena & 2,82 & 2,70 a 2,93 & Satisfacción \\
\hline 12.- Obtengo reconocimiento por mi trabajo & 2,51 & 2,41 a 2,61 & Distensión \\
\hline 13.- La relación con mis jefes es cordial & 3,61 & 3,51 a 3,71 & Relación \\
\hline 14.- Las relaciones con mis compañeros son cordiales & 4,04 & 3,96 a 4,12 & Relación \\
\hline 15.- El sueldo es adecuado & 2,80 & 2,69 a 2,90 & Distensión \\
\hline 16.- Ocupo el puesto que merezco & 4,30 & 4,21 a 4,38 & Satisfacción \\
\hline 17.- Tengo muchas posibilidades de promoción & 1,51 & 1,43 a 1,59 & Distensión \\
\hline 18.- Me falta tiempo para realizar mi trabajo & 2,68 & 2,57 a 2,78 & Presión \\
\hline 19.- Sé lo que se espera de mí en el trabajo & 3,25 & 3,14 a 3,35 & Relación \\
\hline 20.- Creo que mi trabajo es excesivo & 2,85 & 2,76 a 2,95 & Presión \\
\hline 21.- Los problemas de mis compañeros me afectan & 3,60 & 3,51 a 3,70 & Variedad \\
\hline 22.- Con frecuencia siento no estar capacitado para mi trabajo & 1,86 & 1,76 a 1,95 & Adecuación \\
\hline 23.- No tengo recursos suficientes para hacer mi trabajo & 3,17 & 3,09 a 3,26 & Adecuación \\
\hline 24.- La competitividad me causa estrés o tensión & 1,83 & 1,72 a 1,93 & Adecuación \\
\hline
\end{tabular}

Tabla 2: Media de cada uno de los items del cuestionario "Font Roja” y dimensión a la que corresponden.

bal del cuestionario Font Roja, obtuvimos una puntuación media significativamente mayor cuando los encuestados eran: jóvenes $(r=-0,160 ; p<0,001)$, con menos años de experiencia profesional $(r=-0,234$; $\mathrm{p}<0,001)$, con menos años de experiencia penitenciaria $(r=-0,233 ; p<0,001)$, con menor número de internos en su centro de trabajo $(r=-0,217 ; p<0,001)$. Los profesionales más satisfechos laboralmente eran mujeres, con experiencia previa al trabajo en centros penitenciarios, realizaban cursos de formación continuada al menos una vez al año y realizaban consultas programadas de enfermería en sus respectivos centros.

La relación entre las variables que resultaron significativas en el análisis anterior (edad, sexo, experiencia profesional, experiencia penitenciaria, experiencia previa a prisiones, realización de cursos de formación y realización de consultas de enfermería) y las
7 dimensiones del cuestionario de satisfacción laboral se recogen en las Tablas 3 y 4.

El análisis multivariante con regresión lineal no permitió construir un modelo con suficiente capacidad predictiva para la satisfacción global media $\left(\mathrm{R}^{2}=\right.$ $0,146$ y 0,127$)$.

\section{DISCUSIÓN}

De una forma general, la satisfacción laboral de los profesionales de la enfermería penitenciaria, es ligeramente inferior a la de otros profesionales sanitarios, tanto en el ámbito de la atención primaria ${ }^{3,6}{ }^{6} \mathrm{co}-$ mo del ámbito de la atención hospitalaria ${ }^{7,8}$ en nuestro país. El grado de satisfacción es menor, conforme se presenta una mayor edad, una mayor experiencia y una mayor antigüedad, datos que no son de extra- 


\begin{tabular}{|c|c|c|c|c|c|c|c|c|}
\hline & $\begin{array}{c}\text { Satisfacción } \\
\text { laboral }\end{array}$ & $\begin{array}{l}\text { Presión } \\
\text { en el } \\
\text { trabajo }\end{array}$ & $\begin{array}{l}\text { Relaciones } \\
\text { personales }\end{array}$ & $\begin{array}{l}\text { Distensión } \\
\text { en el } \\
\text { trabajo }\end{array}$ & $\begin{array}{c}\text { Adecuación } \\
\text { para el } \\
\text { trabajo }\end{array}$ & $\begin{array}{l}\text { Control } \\
\text { sobre el } \\
\text { trabajo }\end{array}$ & $\begin{array}{l}\text { Variedad } \\
\text { de la } \\
\text { tarea }\end{array}$ & $\begin{array}{l}\text { Índice } \\
\text { Global }\end{array}$ \\
\hline Edad & $-0,233 * \%$ & $-0,285^{* *}$ & $-0,038$ & $-0,114 *$ & 0,090 & $0,128 *$ & 0.000 & $-0,160 \% *$ \\
\hline Años de experiencia profesional & $-0,195 *$ & $-0,348 * *$ & $-0,040$ & $-0,148 * *$ & 0,043 & 0,072 & $-0,075$ & $-0,234 * *$ \\
\hline Años de experiencia penitenciaria & $-0,216 * *$ & $-0,333 *$ & $-0,041$ & $-0,138 \% *$ & 0,076 & 0,079 & $-0,105^{*}$ & $-0,233 \cdots$ \\
\hline Número de internos en el centro & $-0,154 \%$ & $-0,056$ & $-0,144 \%$ & $-0,087$ & $-0,100$ & $-0,197 * * *$ & $-0,062$ & $-0,217 * *$ \\
\hline $\mathrm{N}^{\circ}$ de centros distintos donde ha trabajado & $-0,151 * *$ & $-0,092$ & $-0,007$ & $-0,074$ & 0,044 & 0,092 & $-0,010$ & $-0,074$ \\
\hline
\end{tabular}

Resultado de las pruebas estadísticas de correlación de Pearson. * $\mathrm{p}<0,05 ; * \mathrm{p}<0,001$.

Tabla 3: Relaciones entre las características profesionales cuantitativas y las distintas dimensiones del cuestionario de satisfacción laboral.

\begin{tabular}{|c|c|c|c|c|c|c|c|c|}
\hline & $\begin{array}{l}\text { Satisfacción } \\
\text { laboral }\end{array}$ & $\begin{array}{l}\text { Presión } \\
\text { en el } \\
\text { trabajo }\end{array}$ & $\begin{array}{l}\text { Relaciones } \\
\text { personales }\end{array}$ & $\begin{array}{c}\text { Distensión } \\
\text { laboral }\end{array}$ & $\begin{array}{c}\text { Adecuación } \\
\text { laboral }\end{array}$ & $\begin{array}{c}\text { Control } \\
\text { del } \\
\text { trabajo }\end{array}$ & $\begin{array}{l}\text { Variedad } \\
\text { de la } \\
\text { tarea }\end{array}$ & $\begin{array}{l}\text { Índice } \\
\text { Global }\end{array}$ \\
\hline \multicolumn{9}{|l|}{ Sexo } \\
\hline Mujer & $3,57 *$ & $2,23 *$ & $3,54 *$ & 3,12 & 3,26 & 2,75 & 1,66 & $2,88^{*}$ \\
\hline Hombre & 3,43 & 2,04 & 3,40 & 3,03 & 3,20 & 2,80 & 1,66 & 2,80 \\
\hline \multicolumn{9}{|c|}{ Experiencia Laboral previa } \\
\hline Sí & $3,54 \%$ & 2,17 & $3,50 *$ & 3,09 & 3,24 & 2,76 & 1,67 & $2,85^{*}$ \\
\hline No & 3,22 & 2,00 & 3,31 & 3,07 & 3,13 & 2,90 & 1,50 & 2,73 \\
\hline \multicolumn{9}{|c|}{ Formación continuada } \\
\hline Sí & $3,66 * *$ & 2,21 & 3,51 & $3,14 *$ & 3,26 & 2,76 & $1,75^{*}$ & $2,90 * *$ \\
\hline No & 3,32 & 2,08 & 3,44 & 3,00 & 3,20 & 2,80 & 1,54 & 2,77 \\
\hline \multicolumn{9}{|c|}{ Consultas programadas } \\
\hline Sí & $3,60 *$ & 2,18 & 3,51 & 3,11 & 3,27 & 2,79 & $1,75^{*}$ & $2,89 * *$ \\
\hline No & 3,37 & 2,11 & 3,44 & 3,05 & 3,18 & 2,75 & 1,51 & 2,77 \\
\hline
\end{tabular}

Los resultados de las pruebas estadísticas con dos muestras independientes para variables categóricas son medias. Cuando la diferencia de medias es significativa el valor más alto se muestra con la significación estadística: * $\mathrm{p}<0,01 ; * * \mathrm{p}<0,001$.

Tabla 4: Relaciones entre las características profesionales cualitativas y las distintas dimensiones del cuestionario de satisfacción laboral.

ñar, si tenemos en cuenta, que no existe ninguna forma de promoción o reconocimiento del trabajo realizado en el ámbito penitenciario, a diferencia del resto de ámbitos en los que sí existe una carrera profesional que reconoce esa experiencia y antigüedad de los profesionales de enfermería y que viene regulado por mandato legal en nuestro país. Igualmente, destaca un bajo índice para las dimensiones de monotonía, control de la tarea, o presión en el trabajo, datos que coinciden con algún otro estudio publicado ${ }^{7} \mathrm{y}$ que manifiestan que el trabajo de enfermería en las prisiones, en general, no es un trabajo excesivamente estresante. A pesar de lo anterior, se trata de una actividad sin iniciativa propia del profesional para organizar y dirigir su propio trabajo y bastante rutinario, poderosas razones que contribuyen de una forma manifiesta a que disminuya el índice global de satisfacción laboral. En general, existe una cierta indiferencia por el salario o la competitividad entre los compañeros, se consideran con una buena adecuación para realizar el trabajo, existiendo buenas relaciones personales entre ellos, a diferencia de otros ámbitos, como se manifiesta en algún otro trabajo publicado?

Una posible limitación de este trabajo es que se haya podido cometer un cierto sesgo de selección al ofertar la participación de forma voluntaria, pero creemos que este posible sesgo se minimiza de forma importante al haber tenido unas tasas de participación y respuesta bastante elevadas.

Por otro lado, pensamos que es un trabajo lo suficientemente representativo del colectivo de enfermería penitenciaria de nuestro país, al estar representados prácticamente la totalidad de centros de todas las comunidades autónomas. 
Entre alguna de las principales consecuencias que pueden provocar la insatisfacción laboral, nos encontramos con el aumento del absentismo laboral y el aumento en el número de accidentes laborales, razones estas, por las que los servicios de prevención de las empresas, cada vez más, se interesan por conocer el grado de satisfacción laboral de sus empleados. El índice global de satisfacción encontrado es aceptable, pero en un medio tan peculiar como es el penitenciario con sus características de aislamiento profesional y otras propias de la población atendida, como falta de hábitos saludables, extrema morbilidad de cuadros infecciosos y psiquiátricos, agresividad, etcétera, las administraciones públicas están más obligadas a implementar medidas de soporte para sus profesionales, y que estas sean adecuadas para evitar que éste índice merme y contribuyan a su incremento. Entre ellas son citables:

- Establecimiento de una carrera profesional como forma de incentivar el trabajo de la enfermería en prisiones.

- Planificación y organización de tareas que requieran la participación activa del personal de enfermería como: establecimiento de protocolos de actuación elaborados por los propios profesionales, realización de consultas de enfermería, participación en sesiones clínicas.

- Promover más la formación continuada del personal.

- Promover la participación de enfermería en tareas de investigación.

\section{CORRESPONDENCIA}

Enrique J. Vera-Remartínez.

Centro Penitenciario de Castellón 1.

Carretera de Alcora, km. 10

12071 CASTELLÓN

enriquevera@ono.com

\section{BIBLIOGRAFÍA}

1. Commitee to Design a Strategy for Quality Review and Assurance in Medicare. Medicare. A strategy for quality assurance. Lohr KN editor. Institute of Medicine. Washington, DC: National Academy Press, 1990.

2. Sociedad Española de Médicos de Atención Primaria SEMERGEN [Página principal en Internet] Madrid: Modelos de calidad asistencial en atención primaria. Desarrollo y aplicación de la calidad asistencial en atención primaria. Opinión de la administración. Disponible en: http://www. semergen.es/semergen2/microsites/opinion/opinion5/desarrollo.pdf

3. Sobrequés J, Cebriá J, Segura C, Rodríguez C, Garcia M, Juncosa S. La satisfacción laboral y el desgaste profesional de los médicos de atención primaria. Aten Primaria 2003; 31:227-33.

4. Fernández MI, Moinelo A, Villanueva A, Andrade C, Rivera M, Gómez JM, et al. Satisfacción laboral de los profesionales de atención primaria del área 10 del Insalud de Madrid. Rev Esp Salud Pública 2000; 74:139-47.

5. Aranaz J, Mira J. Cuestionario Font Roja. Un instrumento de medida de la satisfacción en el medio hospitalario. Todo Hosp 1988; 52:63-66.

6. Olivar C, González S, Martínez MM. Factores relacionados con la satisfacción laboral y el desgaste profesional en los médicos de atención primaria de Asturias. Aten Primaria 1999; 24:352-9.

7. López F, Bernal LYC. Satisfacción laboral de los profesionales en un hospital comarcal de Murcia. Rev Calidad Asistencial 2001; 16:243-6.

8. Ignacio E, Córdoba JA, Serrano A, Figueroa E, Moreno J, Ignacio JM. Factores asociados a la satisfacción de los profesionales en los hospitales públicos andaluces. Todo Hosp. 1997; 141:7-12.

9. Anglada N, Ledesma E. Satisfacción de Enfermería de Atención Primaria en las comarcas del Alt y Baix Empordà. Aten Primaria 2000; 25:326-30. 